\title{
Nose-Tip Transition Control by Surface Roughness on a Hypersonic Sphere*
}

\author{
Kiran Joy Irimpan1, Viren Menezes ${ }^{1 \#, ~ K . ~ S r i n i v a s a n ², ~ H a m i d ~ H o s s e i n i ~}{ }^{3 \#}$ \\ ${ }^{1}$ Department of Aerospace Engineering, Indian Institute of Technology Bombay, Mumbai, India \\ ${ }^{2}$ Vikram Sarabhai Space Centre, Thiruvananthapuram, India \\ ${ }^{3}$ Department of Bioelectrics, Institute of Pulsed Power Science, Kumamoto University, Kumamoto, Japan \\ Email: "viren@aero.iitb.ac.in, "hosseini@kumamoto-u.ac.jp
}

How to cite this paper: Irimpan, K.J., Menezes, V., Srinivasan, K. and Hosseini, H. (2018) Nose-Tip Transition Control by Surface Roughness on a Hypersonic Sphere. Journal of Flow Control, Measurement \& Visualization, 6, 125-135.

https://doi.org/10.4236/jfcmv.2018.63011

Received: December 31, 2017

Accepted: April 1, 2018

Published: June 29, 2018

Copyright $\odot 2018$ by authors and Scientific Research Publishing Inc. This work is licensed under the Creative Commons Attribution International License (CC BY 4.0).

http://creativecommons.org/licenses/by/4.0/

\begin{abstract}
The surface heat flux on a $100 \mathrm{~mm}$ diameter hypersonic sphere was reduced through surface roughness on its forebody. The test model was subjected to a hypersonic freestream of Mach 8.8 and Reynolds number 1.98 million/m, in a shock tunnel. Forebody surface heat transfer rates measured on smooth and rough spheres, under the same free-stream conditions, were compared. The comparison of heat flux indicated an overall reduction in surface heating rates on the rough model, which could be attributed to the delayed nose tip transition. The surface roughness on the forebody of the model generated miniature cavities. Stability of the free shear layer over the miniature cavities and entrapment of the destabilizing vortices in the cavities, make the flow over the rough test model more stable than the attached boundary layer over the smooth model, under transitional conditions.
\end{abstract}

\section{Keywords}

Shock Tunnel, Hypersonic, Transition, Surface Roughness, Thermocouple

\section{Introduction}

Hypersonic reentry vehicles have large forebody bluntness to shield from high aerodynamic heating. The large bluntness at hypersonic Mach numbers generates strong bow shock waves due to which the shock layers will have strong entropy layers, which are a region of strong vorticity [1]. Strong entropy layers destabilize the boundary layers, making them susceptible to even small perturbations in the free stream [2]. The entropy layers of reasonable thickness have been

${ }^{\star}$ Part of this paper was presented at 14th International Conference on Fluid Dynamics (ICFD14) in Sendai, Japan, 1-3 Nov. 2017. 
found useful in delaying transition on hypersonic slender blunt cones, where the nose bluntness was moderate [3] [4]. But, a large nose bluntness, which is required for thermal survival of reentry capsules, was found counterproductive as far as transition control was concerned, which was labelled "blunt nose paradox" in literature [1]. The large nose bluntness promoted transition prematurely, despite having a strong favourable pressure gradient around the nose region.

A patch of large roughness, which is of the order of boundary layer thickness on a surface can give rise to a cavity effect, which when subjected to a moderate flow speed can delay transition by virtue of vortex entrapment and persistence in the cavities [5] [6]. The surface with large roughness is physically similar to a wavy surface, which was found effective in suppressing high-frequency instabilities in high-speed flows [7] [8] [9], which delayed transition. The free shear layer due to multiple, short separations over the miniature cavities was found more stable when compared to the attached boundary layer under transition [8] [10]. The multiple, short separations could generate stable separation bubbles in the cavities, which aided the stability of the free shear layer. These stable separation bubbles also damped the acoustic resonances, preventing the growth of instabilities downstream. The shock layer of a hypersonic blunt body could have a large subsonic pocket when the bluntness is large, and the flow speed within the shock layer could be considered moderate. A rough surface in such a flow could delay the boundary layer transition owing to the conditions and reasons stated above.

Reentry vehicles require Thermal Protection Systems (TPS) in addition to a natural heat shield like forebody bluntness, to withstand aerodynamic heating. The thermal protection systems can be classified as active, passive and ablative depending upon their mode of function [11]. An ablative TPS, such as Phenolic Impregnated Carbon Ablator (PICA) and Super Lightweight Ablator (SLA), which is a thin, temperature-sensitive coating on the forebody of the vehicle, is regarded very effective for reentry missions [12] [13] [14]. The ablative TPS evaporates on absorbing heat from the shock layer, leaving behind a roughness on the coated surface [15].

The present study had an objective of investigating the effect of such a surface roughness on the aerothermodynamics of the vehicle. After completing similar investigations on a large angled blunt cone [16], we continued the research on a large sphere (large w.r.t. tunnel test section). Roughness elements to be placed on the model surface were chosen to match their height with the order of the boundary layer thickness, as the roughness of the order of the boundary layer thickness was the most effective in delaying the nose-tip transition [16]. The present phase of investigation comprised surface heat flux measurements on the forebody of the model and the visualization of its flow field, in a hypersonic shock tunnel of Mach 8.8. Heat flux was measured using E-type coaxial thermocouples [17] and the flow visualization was accomplished using a high-speed video camera, aligned with a standard shadowgraph. The study revealed reduction in surface heat flux over a substantial portion of the forebody of the rough 
model, when compared to the smooth model (baseline).

\section{Experimental Method}

\subsection{The Shock Tunnel}

The experiments were performed in the IIT Bombay Shock Tunnel (IITB-ST) [18], with the freestream conditions presented in Table 1. IITB-ST is a standard shock tunnel, operating in the reflected-shock mode. The tunnel can accommodate models of (up to) $100 \mathrm{~mm}$ diameter/width in its test section (dimensions: $300 \times 300 \times 450 \mathrm{~mm}$ ) without blockage. A planar shock wave, generated by bursting a metallic diaphragm in the shock tube, is used to compress and heat the test gas, twice, to obtain a momentary reservoir of high-enthalpy test gas at the entry to a conical, hypersonic nozzle. The nozzle expands the shocked test gas to a hypersonic freestream of Mach $8.8 \pm 2.5 \%$ in the test section. A schematic of the IITB-ST is shown in Figure 1.The shock tube pressure trace nearest to the nozzle $\left(\mathrm{P}_{0}\right)$, and the time-history of the total pressure ratio $\left(\mathrm{P}_{02} / \mathrm{P}_{0}\right)$ at the test location are presented in Figure 2. The steady part of the $\mathrm{P}_{0}$ pressure trace indicates the available test time, while the useful test time is indicated by the stable region of the $\mathrm{P}_{02} / \mathrm{P}_{0}$ trace.

\subsection{Test Model and Instrumentation}

The test model was a sphere of $100 \mathrm{~mm}$ diameter as shown in Figure 3. The forebody of the sphere was roughened by sticking graded grits. Special care was taken to ensure uniformity in the roughness distribution. The average height of the roughness was $1.4 \mathrm{~mm} \pm 15 \%$, which was of the order of the boundary layer thickness (average) on the forebody, estimated in a separate investigation [19]. Seven E-type, co-axial thermocouples were flush-mounted in the surface of the test model along an outward ray from the stagnation point. The arrangement of thermocouples in the model is shown in Figure 3. The thermocouples were fabricated and calibrated in-house [18], which had a sensitivity of $63.6 \pm 1.2 \mu \mathrm{V} /{ }^{\circ} \mathrm{C}$

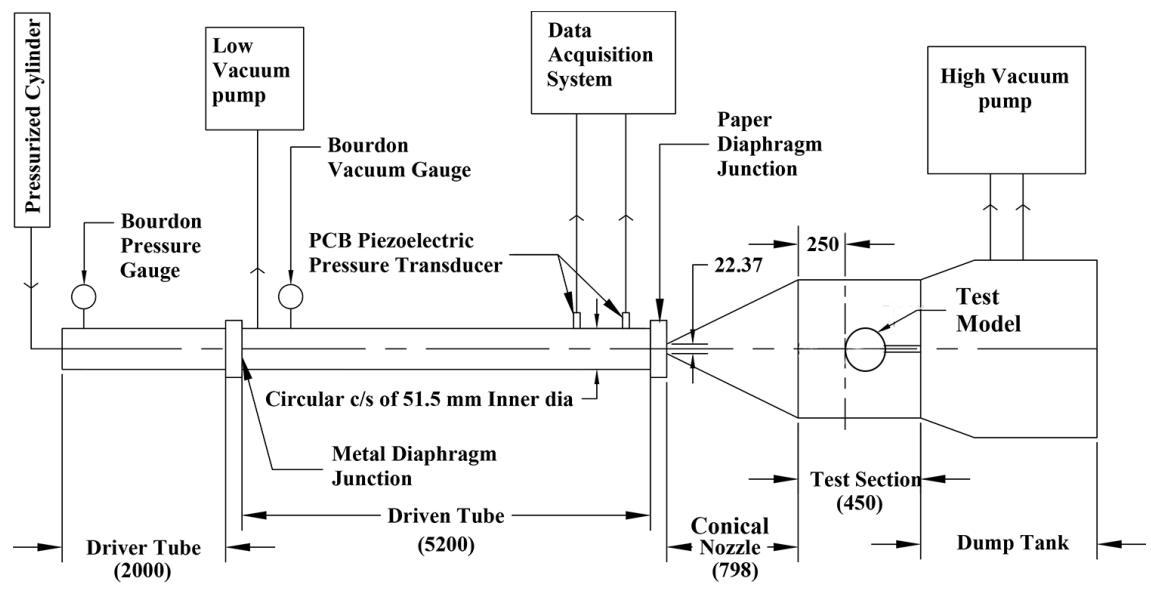

Dimensions in mm

Figure 1. A schematic of the hypersonic shock tunnel, IITB-ST. 
Table 1. Freestream conditions in IITB-ST for the present set of experiments.

\begin{tabular}{ccccc}
\hline Mach no. & $\mathrm{P}_{\infty}(\mathrm{Pa})$ & $\mathrm{T}_{\infty}(\mathrm{K})$ & $\mathrm{H}_{0}(\mathrm{MJ} / \mathrm{kg})$ & $\mathrm{Re} / \mathrm{m}\left(\times 10^{6}\right)$ \\
\hline $8.8 \pm 0.2$ & $112 \pm 5$ & $65 \pm 2$ & $1.12 \pm 0.4$ & $1.98 \pm 0.02$ \\
\hline
\end{tabular}
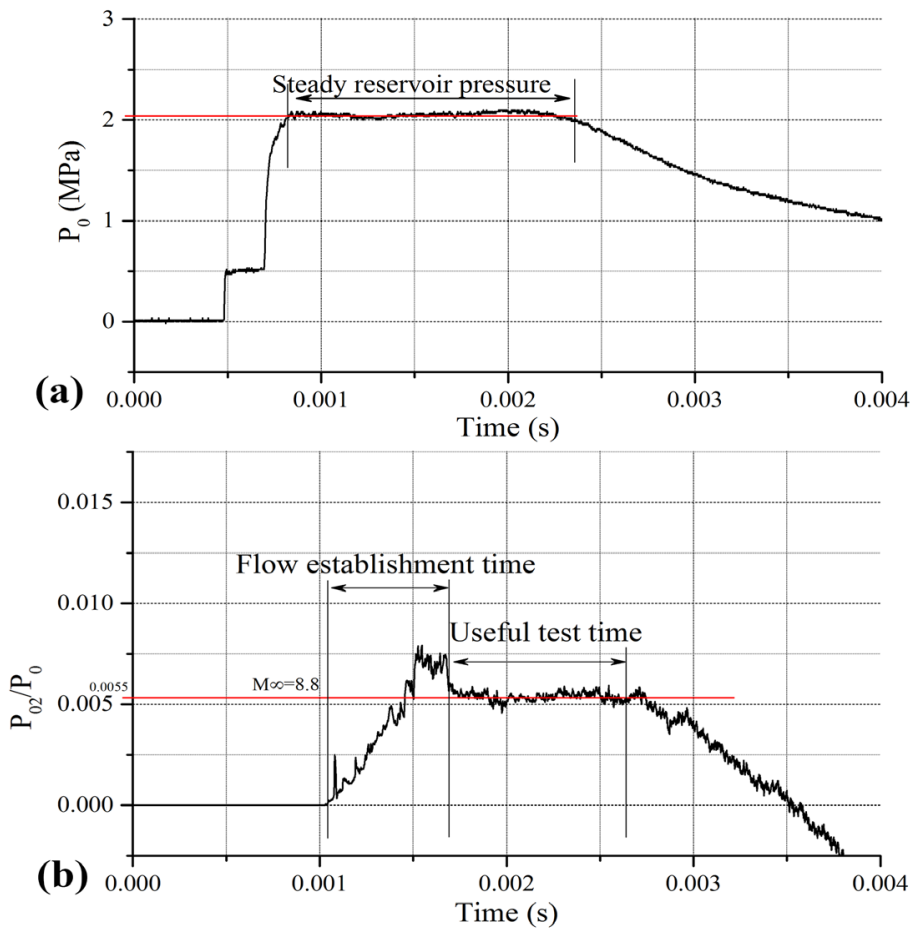

Figure 2. Pressure-time history in the nozzle reservoir (a) and at the test location (b). PCB-Piezotronics (USA) pressure transducers, models 102A and 102A08 were used for (a) \& (b), respectively.

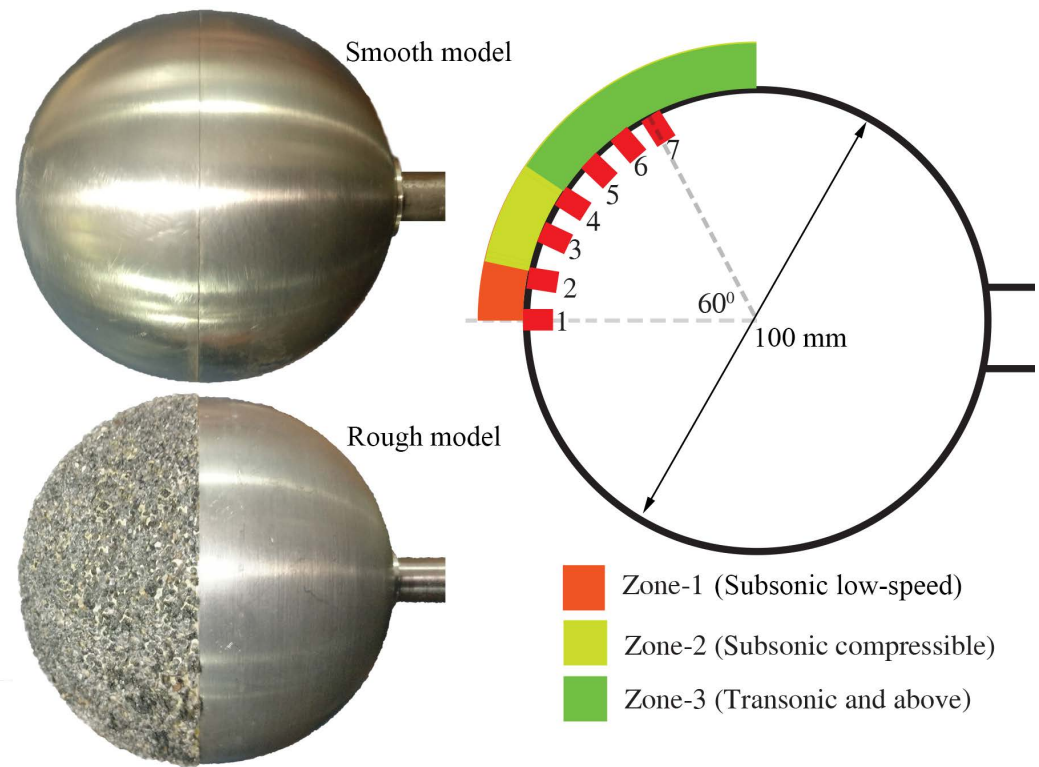

Thermocouples are marked red and numbered 1-7

Figure 3. The test model. 
and an effective thermal product range of $5857-12,306 \mathrm{Jm}^{-2} \cdot \mathrm{K}^{-1} \cdot \mathrm{s}^{-1 / 2}$. The thermocouples were instrumented with INA128 instrumentation amplifiers with a gain factor of 500 and a peak operating frequency of $40 \mathrm{kHz}$. In the rough surface, the sensing junctions of the thermocouples were at half the roughness height from the model surface. The sensor placement in the rough model is presented in Figure 4. This arrangement was expected to capture the effect of miniature cavities in the surface. The smooth model (without grits) was the baseline of the investigation.

The shock tunnel is equipped with an 8-inch Z-type Schlieren system [20] for visualization of the flow fields over test models. The major components of the Schlieren system are the 8-inch parabolic mirrors, a continuous light source of 150 Watt (LS-150, xenon-short-arc lamp, Abet Technologies, USA) and a charge-coupled-device video camera (Phantom v710-1113, Vision Research Inc., USA). The flow visualization over the sphere was carried out with a sampling rate and a spatial resolution of 13 kilo frames per second and $608 \times 800$ pixels, respectively. The camera was triggered externally using an amplified output of the shock tube pressure transducer on the arrival of the incident shock wave. The Phantom camera used a variable focal length lens of Nikon make (AF Nikkor 80 - $200 \mathrm{~mm} \mathrm{F/2.8D} \mathrm{ED,} \mathrm{Japan).} \mathrm{The} \mathrm{Schlieren} \mathrm{was} \mathrm{used} \mathrm{in} \mathrm{the} \mathrm{shadowgraph}$ mode for the present investigation.

The output of the sensors in the test model was acquired on a data acquisition system, equipped with NI-PCI-6115 S series data cards (National Instruments Corporation, USA), at a sampling rate of $1 \mathrm{MS} / \mathrm{s}$. The acquired signals were post-processed using a $4^{\text {th }}$ order IIR low-pass filter (cut-off frequency of $10 \mathrm{kHz}$ ) to eliminate the high-frequency spurious noise.

\subsection{Thermocouple Data Reduction Methodology}

The surface heat-flux was reduced from the acquired temperature-time history signals of the flush mounted thermocouples based on the methodology proposed
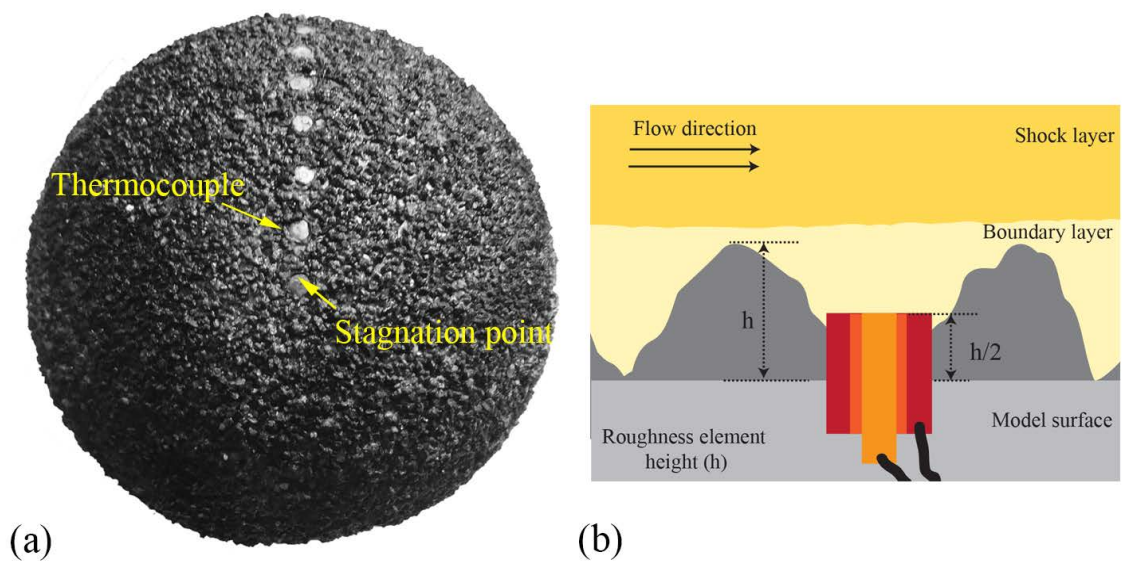

(b)

Figure 4. Sensor placement in the rough test model for heat flux measurement. (a) Photograph of the model forebody with the sensors; (b) A schematic indicating the level of the sensor w.r.t. the roughness element. 
by Cook \& Felderman, which is on the assumption of 1-D heat conduction in a semi-infinite slab [21]. A 1-D heat conduction model [22], represented by Equation (1), was used to obtain such an unsteady heat flux into the sensing junction of the thermocouple.

$$
Q(t)=\frac{\beta}{\sqrt{\pi} \alpha}\left[\frac{E(t)}{\sqrt{t}}+\frac{1}{2} \int_{0}^{t} \frac{E(t)-E(\tau)}{(t-\tau)^{3 / 2}} d \tau\right]
$$

Equation (1) was numerically processed using a piece-wise linear function for $E(\tau)$, as expressed in Equation (2).

$$
E(\tau)=E\left(t_{i-1}\right)+\frac{E\left(t_{i}\right)-E\left(t_{i-1}\right)}{\Delta t}\left(\tau-t_{i-1}\right)
$$

where, $t_{i-1} \leq \tau \leq t_{i}$ and $i=1,2,3, \cdots, n$. The Equation (2) was substituted into Equation (1) and was integrated to obtain the following expression:

$Q(t)=\frac{\beta}{\sqrt{\pi} \alpha}\left[\frac{E\left(t_{n}\right)}{\sqrt{t_{n}}}+\sum_{i=1}^{n-1}\left\{\frac{E\left(t_{n}\right)-E\left(t_{i}\right)}{\sqrt{t_{n}-t_{i}}}-\frac{E\left(t_{n}\right)-E\left(t_{i-1}\right)}{\sqrt{t_{n}-t_{i-1}}}+2 \frac{E\left(t_{i}\right)-E\left(t_{i-1}\right)}{\sqrt{t_{n}-t_{i}}+\sqrt{t_{n}-t_{i-1}}}\right\}+\frac{E\left(t_{n}\right)-E\left(t_{n-1}\right)}{\sqrt{\Delta t}}\right]$

Equation (3) was programmed to obtain the heat flux-time histories from the temperature-time histories of the thermocouples. The heat flux signals were time-averaged over the useful test time of the shock tunnel for reading.

\section{Results and Discussion}

Figure 5 presents representative heat flux signals on the forebody of the sphere, in the three zones (as marked in Figure 3). The signals are of smooth and rough surfaces for the same freestream conditions. The zone-1 had a low-speed (subsonic) flow, zone-2: compressible-subsonic flow, and zone-3: transonic and supersonic flows. The heat flux at each thermocouple location was normalized w.r.t. the stagnation point heat flux for that particular shot, and was plotted w.r.t. the non-dimensional wetted length along the model surface, as in Figure 6. The normalizing stagnation point heat fluxes were 20.1 and $19.8 \mathrm{~W} / \mathrm{cm}^{2}$ for the
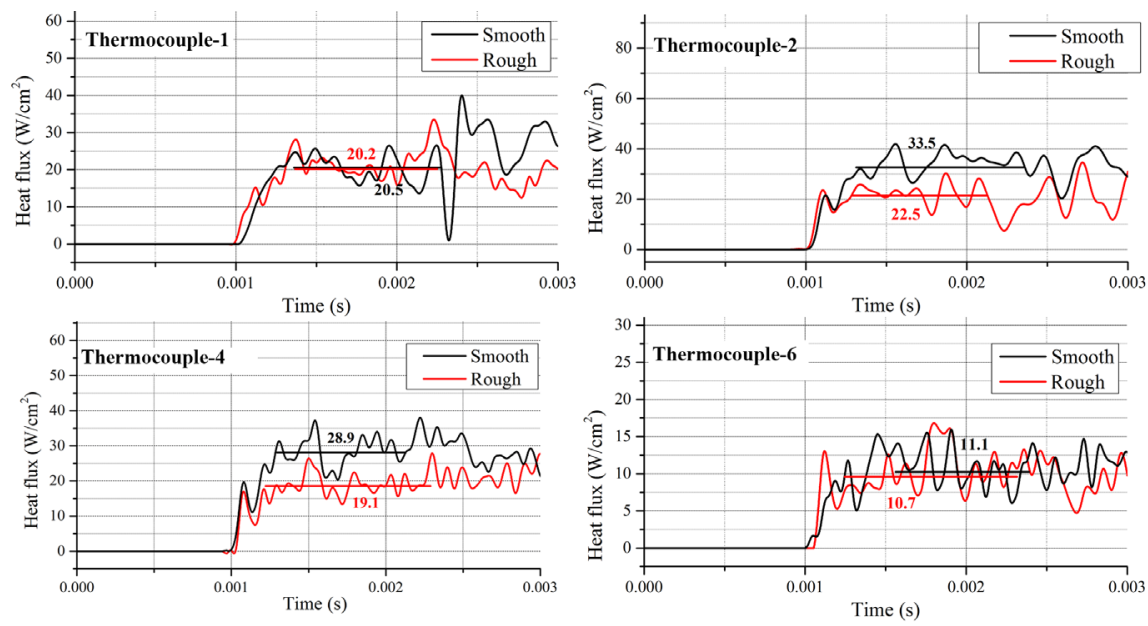

Figure 5. Heatflux-time history in different zones of the model forebody. 


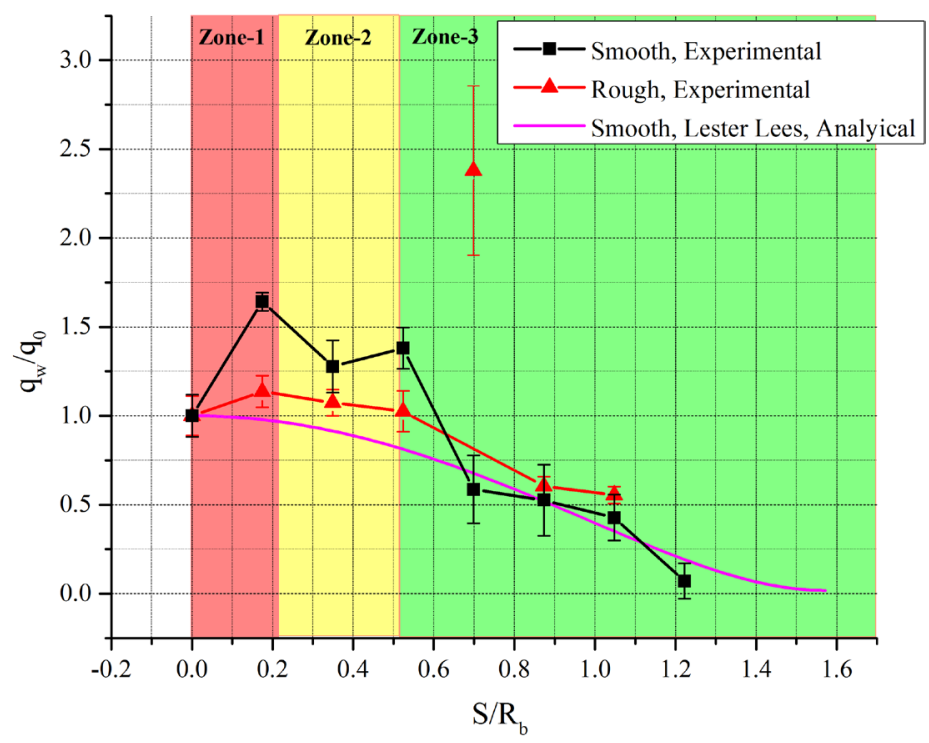

Figure 6. Heatflux distribution on the sphere, along an outward ray on the forebody.

smooth and the rough models, respectively. The measured heat-flux distribution over the smooth sphere indicated a transitional trend as seen in the plot in Figure 6. A considerable reduction in heat flux was observed in zone-2, on a rough surface, where the trend of distribution was leaning towards laminar. An analytical, laminar calculation of the heat flux based on Lester Lees formulation [23] for the smooth sphere is included in Figure 6. A comparison between the experimental and the analytical heat flux distributions confirms the tendency of the rough surface to delay the transition in zone-2. An overall reduction of $16 \%$ in the forebody surface heat flux was observed on the rough sphere when compared to the smooth. The zone- 2 is subsonic-compressible in nature and the rough surface appears to have reacted to this feature favourably. Delay in transition brought about by the surface roughness is the reason for the reduced heat flux in zone-2 [6]. The visualized flow field images of the models are presented in Figure 7. The images show unsteadiness in the flow field of the smooth sphere while it is damped in the flow field of the rough sphere (around zones $1 \& 2$ ). The flow field images corroborate the observed trends of the measured heat flux distribution.

The large-scale roughness generated a multiple-cavity effect on the forebody of the sphere. Under moderate velocities, such as in zone-2, the roughness cavities could engulf the destabilizing entropy layer in the form of trapped large eddies thereby damping the associated instabilities. The destabilizing factor of the entropy layer was isolated, damped and confined to these cavities till the vortices started shedding, and the separated shear layer over the cavities was more stable than its transitional, attached counterpart [7]; hence, the transition was delayed. The destabilization was expected to appear once the vortices started shedding from the cavities due to high speed of the flow, which is lightly visible in Figure 6 (zone-3). Figure 8 depicts the above description of multiple-cavity effect and vortex dynamics. 


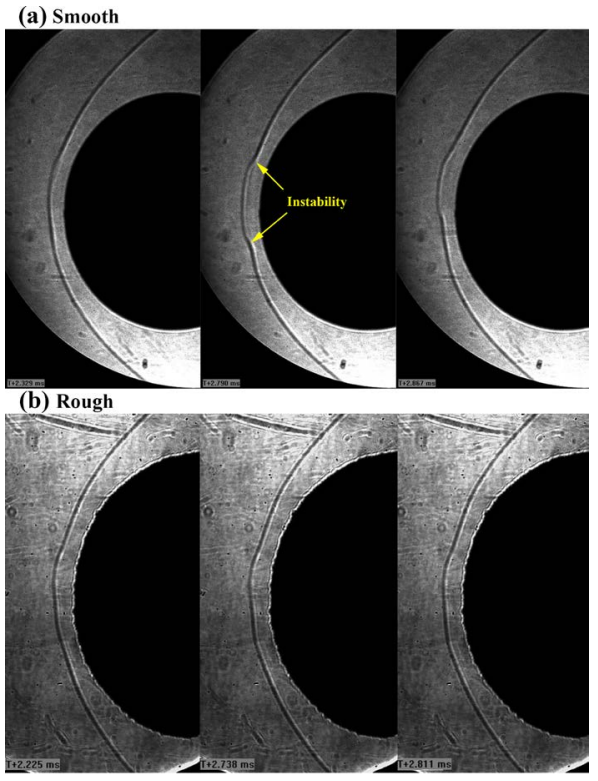

Figure 7. Visualized flow-fields of the smooth (a) and rough (b) spheres.
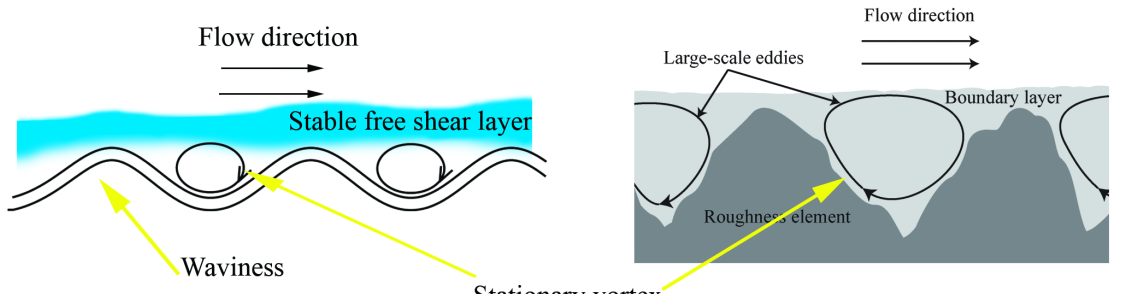

(a) Wavy surface

(b) Rough surface in zone-2

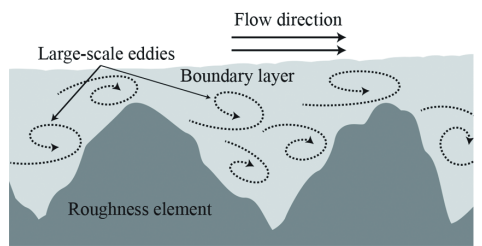

(c) Rough surface in zone-3 (vortex shedding)

Figure 8. Illustration of the multiple-cavity effect and vortex dynamics over a rough surface.

The results presented in Figure 6 encourage the use of surface roughness to delay transition and alleviate the transition-linked detriments, such as skin friction and heat transfer. The study indicates that the remnants of ablative TPS that create a surface roughness may not be detrimental to reentry capsules. The study also revealed that the roughness height, which is of the order of the boundary layer thickness on a surface, is suited to delay the transition.

\section{Concluding Remarks}

Effect of surface roughness on wall heating rates of a $100 \mathrm{~mm}$ diameter sphere was investigated in a hypersonic freestream of Mach 8.8. The roughness on the 
forebody of the sphere was found to reduce surface heat flux in a zone with moderate flow velocity. The reason could be attributed to vortex persistence in the roughness cavities and delayed-transition. Qualitative flow visualization on the models corroborated the measured heat flux data. The study has relevance to ablative Thermal Protection Systems (TPS) of reentry capsules.

\section{Acknowledgements}

This work was funded by the ISRO-IITB-Space Technology Cell, IIT Bombay (Grant\# 15ISROC010).

\section{References}

[1] Anderson Jr., J.D. (2006) Hypersonic and High-Temperature Gas Dynamics. 2nd Edition, American Institute of Aeronautics and Astronautics, Reston, VA. https://doi.org/10.2514/4.861956

[2] Fedorov, A.V. (1990) Instability of the Entropy Layer on a Blunt Plate in Supersonic Gas Flow. Journal of Applied Mechanics and Technical Physics, 31, 722-728. https://doi.org/10.1007/BF00852446

[3] Stetson, K. (1983) Nosetip Bluntness Effects on Cone Frustum Boundary Layer Transition in Hypersonic Flow. 16th Fluid Plasmadynamics Conference, Massachusetts, 12-14 July 1983. https://doi.org/10.2514/6.1983-1763

[4] Ericsson, L.E. (1988) Effect of Nose Bluntness and Cone Angle on Slender-Vehicle Transition. AIAA Journal, 10, 1168-1174. https://doi.org/10.2514/3.10024

[5] Balle, G.J. and Breidenthal, R.E. (2002) Stationary Vortices and Persistent Turbulence in Karman Grooves. Journal of Turbululence, 3.

[6] Fujii, K. (2006) Experiment of the Two-Dimensional Roughness Effect on Hypersonic Boundary-Layer Transition. Journal of Spacecraft and Rockets, 43, 731-738. https://doi.org/10.2514/1.17860

[7] Bountin, D., Chimitov, T., Maslov, A., Novikov, A., Egorov, I., Fedorov, A. and Utyuzhnikov, S. (2013) Stabilization of a Hypersonic Boundary Layer Using a Wavy Surface. AIAA Journal, 51, 1203-1210. https://doi.org/10.2514/1.J052044

[8] Egorov, I.V., Novikov, A.V. and Federov, A.V. (2010) Direct Numerical Simulation of supersonic Boundary Layer Stabilization Using Grooved Wavy Surface. 48th AIAA Aerospace Sciences Meeting Including the New Horizons Forum and Aerospace Exposition, Florida, 4-7 January 2010, Orlando, AIAA 2010-1245.

[9] Zhou, Y., Liu, W., Chai, Z. and Yang, X. (2017) Numerical Simulation of Wavy Surface Effect on the Stability of a Hypersonic Boundary Layer. Acta Astronautica, 140, 485-496. https://doi.org/10.1016/j.actaastro.2017.08.018

[10] Egorov, I.V., Novikov, A.V. and Fedorov, A.V. (2006) Numerical Modeling of the Disturbances of the Separated Flow in a Rounded Compression Corner. Fluid Dynamics, 41, 521-530. https://doi.org/10.1007/s10697-006-0070-7

[11] Venkatapathy, E., Laub, B., Hartman, G J., Arnold, J.O., Wright, M.J. and Allen, G.A. (2009) Thermal Protection System Development, Testing, and Qualification for Atmospheric Probes and Sample Return Missions. Examples for Saturn, Titan and Stardust-Type Sample Return. Advance in Space Research, 44, 138-150. https://doi.org/10.1016/j.asr.2008.12.023

[12] Strauss, E.L. (1967) Superlight Ablative Systems for Mars Lander Thermal Protection. Journal of Spacecraft, 4, 1304-1309. https://doi.org/10.2514/3.29076 
[13] Tran, H.K., Johnson, C.E., Rasky, D.J., Hui, F.C.L., Chen, Y.K. and Hsu, M.-T. (1996) Phenolic Impregnated Carbon Ablators (PICA) for Discovery Class Missions. 31 st AIAA Thermophysics Conference, New Orleans, 17-20 June 1996, AIAA Paper 96-1911.

[14] Milos, F.S. and Chen, Y.-K. (2009) Ablation and Thermal Response Property Model Validation for Phenolic Impregnated Carbon Ablator. 47th AIAA Aerospace Sciences Meeting Including the New Horizons Forum and Aerospace Exposition, Orlando, 5-8 January 2009, AIAA 2009-262. https://doi.org/10.2514/6.2009-262

[15] Reda, D.C., Wilder, M.C. and Prabhu, D.K. (2011) Transition Experiments on Slightly Blunted Cones with Distributed Roughness in Hypersonic Flight. 41st AIAA Fluid Dynamics Conference and Exhibit, Hawaii, 27-30 June 2011, AIAA 2011-3417. https://doi.org/10.2514/6.2011-3417

[16] Irimpan, K.J. and Menezes, V. (2015) Effect of Surface Roughness on the Heating Rates of Reentry Vehicles. 12 th International Conference on Flow Dynamics, Sendai, 27-29 October 2015, 468-469.

[17] Menezes, V. and Bhat, S. (2010) A Coaxial Thermocouple for Shock Tunnel Applications. Review of Scientific Instruments, 81, Article ID: 104905.

https://doi.org/10.1063/1.3494605

[18] Irimpan, K.J., Mannil, N., Arya, H. and Menezes, V. (2015) Performance Evaluation of Coaxial Thermocouple against Platinum Thin Film Gauge for Heat Flux Measurement in Shock Tunnel. Measurement, 61, 291-298. https://doi.org/10.1016/j.measurement.2014.10.056

[19] Kumar, D.R. (2015) Effect of Size on the Shock Standoff Distance and the Heat Transfer Rates of Hypersonic Blunt Bodies. M-Tech Thesis, IIT Bombay, Mumbai.

[20] Talsaniya, H.M., Menezes, V., Nithin, B. and Kulkarni, V. (2015) Visualization of Incipient Flow Separation Condition on Hypersonic Shock-Boundary Layer Interaction. Journal of Visualisation, 18, 611-618. https://doi.org/10.1007/s12650-015-0274-1

[21] Cook, W.J. and Felderman, E.J. (1966) Reduction of Data from Thin-Film Heat-Transfer Gages: A Concise Numerical Technique. AIAA Journal, 4, 561-562. https://doi.org/10.2514/3.3486

[22] Hollis, B.R. (1995) User's Manual for the One-Dimensional Hypersonic Experimental Data Reduction Code. NASA Contractor Report 4691.

[23] Lees, L. (1956) Laminar Heat Transfer over Blunt-Nosed Bodies at Hypersonic Flight Speeds. Journal of Jet Propulsion, 26, 259-269. https://doi.org/10.2514/8.6977 


\section{Nomenclature}

$E(t)$ : time dependent voltage

$\mathrm{H}_{0}$ : total enthalpy

$\mathrm{P}_{0}$ : reservoir pressure

$\mathrm{P}_{02}$ : pitot pressure

$\mathrm{P}_{\infty}$ : static pressure

$\mathrm{Q}(\mathrm{t})$ : unsteady heat flux

$\mathrm{R}_{\mathrm{b}}$ : base radius

Re: Reynolds number

$S$ : wetted length

$\mathrm{T}_{\infty}$ : static temperature

t: time

w.r.t.: with respect to

$\alpha$ : thermocouple sensitivity

$\beta$ : effective thermal product

$\tau$. time variable 